\title{
Liderazgo y cultura en las instituciones de educación superior
}

Leadership and culture in higher education institutions

Recibido el 02 de agosto del 2017, aceptado el 12 de octubre del 2017

Asencios Trujillo, Lida Violeta lida_asencios@yahoo.com Universidad Nacional Enrique Guzmán y Valle - Perú ORCID: 0000-0001-8834-8084
Para referenciar este artículo: Asencios, L.V. (2017). Liderazgo y cultura en las instituciones de educación superior. ConCiencia EPG, 2(2), 67-70.

\section{Resumen}

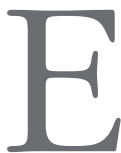

1 artículo se centra en la descripción sobre liderazgo educativo en instituciones de educación superior, y más específicamente, sobre las acciones que se realizan como la gestión total, la planificación estratégica, los beneficios de un liderazgo efectivo, como factor decisivo para la eficacia en el funcionamiento de las instituciones educativas. Se analiza el concepto de liderazgo educativo, como proceso complejo que requiere la mayor flexibilidad y equivalencia entre la planificación, la organización y, sobre todo, el control de actividades y procesos. Finalmente se hace mención a las acciones que realiza el docente como un líder educativo haciendo mención de algunos estudios que demuestran que la calidad docente es el factor de mayor impacto en el aprendizaje de los estudiantes. Ante ello, es necesario la creación de nuevos líderes en la docencia para que provoque nuevas formas de aprendizaje en todos los estudiantes del medio.

Palabras clave: liderazgo, educación, liderazgo docente, cultura institucional

\section{Summary}

The article focuses on the description of educational leadership in higher education institutions, and more specifically, on the actions that are carried out such as total management, strategic planning, the benefits of effective leadership, as a decisive factor for effectiveness in the operation of educational institutions. The concept of educational leadership is analyzed as a complex process that requires the greatest flexibility and equivalence between planning, organization and, above all, the control of activities and processes. Finally, a mention of the actions carried out by the teacher as an educational leader is made, mentioning some studies that show that teacher quality is the factor with the greatest impact 
on student learning. Given this, it is necessary to create new leaders in teaching to provoke new forms of learning in all students of the environment.

Keywords: leadership, education, teacher leadership, institutional culture

\section{Introducción}

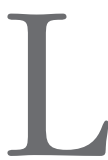

a necesidad de gestionar en las complejidades de nuestro sistema educativo y lograr los objetivos de las instituciones tiene como punto de partida al líder. Según Robinson (2010) la mejora en los resultados de aprendizaje de los estudiantes está fuertemente ligada al liderazgo. Ulloa, Nail, Castro y Muñoz (2012) sostienen que, un liderazgo efectivo del director o directora lleva al establecimiento de metas y expectativas de aprendizajes que se comparten con los docentes, además, se basa en la planificación, coordinación y evaluación de la enseñanza, del currículo y, sobre todo de la promoción y participación en el aprendizaje y desarrollo de los docentes. Ante ello, es la obligación de las instituciones de educación superior garantizar que todos sus estudiantes, logren los aprendizajes necesarios aprovechando las oportunidades de una sociedad del conocimiento y una sociedad red.

Investigaciones realizadas con la finalidad de conocer las implicancias del liderazgo en la buena gestión y el éxito académico (Abreo, 2015; Gutiérrez y Castillo, 2015; Horn y Marfan, 2010; Leithwood, 2009; Pinto, Galdames y Rodriguez, 2010; Rivera, 2015) señalan que el liderazgo es fundamental en las instituciones educativas debido a que tiene una función específica, un propósito y una dirección. El rol del liderazgo del director o directora y también de los docentes, tiene un efecto demostrable en el desempeño académico de sus estudiantes.

Si la influencia del liderazgo es tan importante en distintos aspectos de la organización, nunca en el Perú ni en cualquier parte del mundo se habló tanto del liderazgo efectivo, por la carencia que existe a partir de la crisis educativa. Manzano, Monimó y Carreri (2013) afirman que "Este indicador se considera fundamental puesto que el liderazgo no es observable a simple vista, sino que destila de las acciones, de unas estrategias, que evidentemente persiguen la consecución de unos objetivos a corto, medio o largo plazo" (p. 294). En ese sentido, el análisis sobre el liderazgo es necesario dado que afectan al hecho educativo que configuran y modelan en nuestro Sistema Nacional.

\section{Concepto de liderazgo educativo}

Cuando se hace un repaso sobre esta variable, se encuentra que el concepto "liderazgo educativo" es investigado con bastante amplitud en contextos de educación básica, sin embargo, en educación superior existen estudios esporádicos que dan cuenta de la carencia de evidencias para sustentar su importancia, por ello, se describe este concepto. Según Rivera (2015, p. 9):

El liderazgo educativo es un término que se aplica a los administradores educativos que se esfuerzan por crear un cambio positivo en la política y los procesos educativos. Esos esfuerzos van dirigidos al fortalecimiento de los aprendizajes de los estudiantes, a la equiparación de la facultad y al personal no docente para lograr las metas y los objetivos, tomando como inspiración la misión institucional.

El liderazgo es un proceso complejo que requiere la mayor flexibilidad y equivalencia entre la planificación, la organización y, sobre todo, el control de actividades y procesos. Ante ello, hay una necesidad de desarrollar valores, principios y actitudes dirigidas a la creación de una cultura organizacional fundada en el cambio y en la participación (Lashway, 2003). Además, es imprescindible que conozca las características personales que debe reunir para cumplir su función y, sobre todo, poder auto identificarse con ellas. De esta manera, podrá tener una idea más clara de lo que ofrece en su función de líder y podrá decidir fortalecerse con las teorías emergentes. En 
una sociedad red, es importante la identificación a nivel internacional del liderazgo educativo como una de las principales variables que inciden en el desempeño del estudiante, de todo el sistema y la constatación de una agenda que plantea la innovación de la educación superior en un plazo determinado.

\section{Liderazgo docente}

En los niveles superiores, el liderazgo del docente es clave, investigaciones previas sobre eficacia educativa demuestra que la calidad docente es el factor de mayor impacto en el aprendizaje (Contreras, 2016). Para ello, es necesario una formación adecuada del docente, la preparación para ser líder. Según Rivera (2015), el profesorado, su potencial de liderazgo y el liderazgo pedagógico conforman una nueva noción integral de institución que tiene como máxima servir al estudiante y su aprendizaje, al desarrollo humano y al desarrollo profesional.

Además de la formación pedagógica y el desarrollo profesional es importante el manejo de emociones, Goleman (2013) sostiene que "los modelos de competencia para el liderazgo suelen estar compuesto entre el $80 \%$ y el $100 \%$ por capacidades basadas en la inteligencia emocional" (p. 12). Los docentes con un alto nivel de liderazgo y que influyen de manera positiva en sus estudiantes, son aquellos que mejor inteligencia emocional han desarrollado, tienen una mejor comprensión de sí mismo y del otro, muestran una mejor empatía, son más receptivos $\mathrm{y}$ tienen mejor performance frente al otro.

Por ello, es necesario, tal como señala Gutierrez y Castillo (2015) "que todo docente tiene el deber de desarrollar y mejorar el manejo de sus emociones para brindar con eficiencia servicios docentes y con calidad humana" ( $p$. 33). Según comenta LLinás, (2003) las emociones al igual que los pensamientos, son estados funcionales del cerebro porque allí se genera nuestro "yo" la conciencia de nosotros mismos y del otro, y, además, en el manejo apropiado de las emociones, esta, la autogestión, la conciencia social, y la gestión de las relaciones (Goleman, 2012).

En consecuencia, si se quiere mejores instituciones educativas, mejores estudiantes, mejores profesionales y mejores ciudadanos, es esencial que profesores y directores sean debidamente capacitados, empoderados, y que tomen conciencia de su rol protagónico en dichos procesos (Rivera, 2015). Debido a que la sociedad del conocimiento ha relegado los paradigmas de aprendizaje tradicionales, memorísticos y deterministas, siendo en la actualidad demandadas competencias reflexivas, analíticas e interpretativas, que se basan en el razonamiento crítico-constructivo, generan y aprovechan espacios de innovación y optimización, así como formas de trabajo cooperativas y participativas (Contreras, 2016). Ante ellos, la didáctica también juega un rol protagónico, constituye la parte técnica de la pedagogía, que se encarga de dotar de métodos y técnicas al proceso de enseñanza-aprendizaje antes, durante y después de la realización del trabajo docente y desde la dirección, con nuevas políticas y nuevos currículos hay que mejorarlos.

\section{Conclusiones}

Cuando se habla de liderazgo, se hace referencia a una relación de influencia entre los líderes y sus seguidores, en la cual se pretende compartir resultados que se hayan planteado como metas u objetivos. En este caso, los directores son los encargados de solucionar problemáticas externas a los procesos de enseñanza aprendizajes de los estudiantes y le dan mayor importancia en los procesos de gestión. Estudios como los de Ulloa, Nail, Castro y Muñoz (2012) sugieren que la efectividad académica y la gestión adecuada de los recursos de una institución demuestran que las autoridades de este tipo de instituciones tienen una mirada diferente a las de las instituciones clásicas. Las preocupaciones en este lugar son esencialmente pedagógico curriculares y asumen las responsabilidades de ello. 


\section{Referencias}

Abreo, J.P. (2015). El liderazgo educativo en escenarios de autonomía administrativa: un estudio de caso (Tesis de doctorado). Universidad de Puerto Rico, Rio piedras, Puerto Rico.

Contreras, T. (2016). Liderazgo pedagógico, liderazgo docente y su papel en la mejora de la escuela: una aproximación teórica. Propósitos y Representaciones, 4(2), 231-284. Doi: http://dx.doi.org/10.20511/pyr2016. v4n2.122.

Goleman, D. (2012). La inteligencia emocional: nuevos descubrimientos. Barcelona: Ediciones B. S.A.

Goleman, D. (2013). Liderazgo: el poder de la inteligencia emocional. Barcelona: Ediciones B. S.A.

Gutiérrez, A. y Castillo, J. M. (2015). Docencia y liderazgo en las Ciencias de la Información Documental: un acercamiento. Revista Interamericana de Bibliotecología, 38(1), 2740. Recuperado de http://www.scielo.cl/pdf/ estped/v38n1/art07.pdf

Horn, A. y Marfan, J. (2010). Relación entre liderazgo educativo y desempeño escolar: revisión de la investigación en Chile. Psicoperspectivas individuo y sociedad, 9(2), 82-104.

Lashway, L. (2003). Role of the school leader: Clearinghouse on educational policy and management. OR: College of Education/ University of Oregon.
Leithwood, K, (2009). ¿Cómo liderar nuestras escuelas? Aportes desde la investigación. Santiago: Salesianos Impresores.

LLinás, R. (2003). El cerebro y el mito del yo. Bogotá: Norma.

Manzano, J.M., Monimó, J.M. y Carreri, J. (2013). La planificación estratégica, un indicador sobre el liderazgo pedagógico. Teoría de la educación. Educación y cultura en la sociedad de la información, 14(2), 292315. Recuperado de http://campus.usal. es/ revistas_trabajo/index.php/revistatesi/ article/view/10225/10633.

Pinto, V., Galdames, S. y Rodriguez, S. (2010). Aprendizajes y desafíos para la formación de líderes intermedios de organizaciones educativas. Psicoperspectivas individuo y sociedad, 9(2), 136-157.

Rivera, R. A. (2015). El concepto del liderazgo educativo desde la experiencia de directores de departamento de uno de los recintos de la universidad de Puerto Rico (Tesis de doctorado). Universidad de Puerto Rico, Rio piedras, Puerto Rico.

Robinson, V. (2010). Del Liderazgo al resultado de los estudiantes. Recuperado de http:// www.educarchile.cl

Ulloa, J., Nail, O., Castro, A. y Muñoz, M. (2012). Problemas de gestión asociados al liderazgo como función educativa. Estudios pedagógicos, XXXVIII(1), 121-129. Recuperado de http://www.scielo.cl/pdf/ estped/v38n1/art07.pdf. 